\title{
Cubic Silsesquioxanes as a Green, High-Performance Mold Material for Nanoimprint Lithography
}

\author{
Hyun Wook Ro,* Vera Popova, Lei Chen, Aaron M. Forster, Yifu Ding, Kyle J. Alvine, \\ Dave J. Krug, Richard M. Laine, and Christopher L. Soles*
}

Optical lithography deep in the UV spectrum is the predominate route for high-resolution, high-volume nanoscale pattering. However, state-of-the-art optical lithography tools are exceedingly expensive and this places serious limitations on the applications, technical sectors, and markets where highresolution patterning can be implemented. To date the only substantial market for high-end optical lithography tools has been semiconductor fabrication. Nanoimprint lithography (NIL) has recently emerged as an alternative to optical lithography and combines the potential of sub-five-nanometer patterning resolution with the low cost and simplicity of a stamping process. ${ }^{[1-4]}$ This has led to significant efforts to implement NIL methods, not only for semiconductor logic devices, but also in fields as diverse as the direct patterning of interlayer dielectrics (ILDs) for back-end-of-line (BEOL) interconnect structures, ${ }^{[5-7]}$ bitpatterned magnetic media for data storage, ${ }^{[8,9]}$ and high-brightness light-emitting diodes (LEDs). ${ }^{[10]}$ Some of these are new areas where nanoscale patterning has previously not been considered, and are made possible here by the low cost and simplicity of the NIL stamping processes.

NIL and other stamping processes are direct write $(1 \times)$ technologies. There is a one-to-one correlation between the features in the mold and the imprint, meaning that high-quality imprint molds are critical. In fact, one of the biggest impediments to

Dr. H. W. Ro, Dr. Y. Ding, Dr. K. J. Alvine, Dr. C. L. Soles Polymers Division

National Institute of Standards and Technology (NIST)

100 Bureau Dr., Mail Stop 8541

Gaithersburg, MD 20899, USA

E-mail: hyun.ro@nist.gov csoles@nist.gov

V. Popova, D. J. Krug

Mayaterials Inc., 661 Airport Blvd. Suite 1

Ann Arbor, MI 47108, USA

Dr. L. Chen

Center for Nanoscale Science and Technology

National Institute of Standards and Technology (NIST)

100 Bureau Dr., Mail Stop 6201

Gaithersburg, MD 20899, USA

Dr. A. M. Forster

Materials and Construction Research Division

National Institute of Standards and Technology (NIST)

100 Bureau Dr., Mail Stop 8615

Gaithersburg, MD 20899, USA

Prof. R. M. Laine

Department of Materials Science and Engineering

University of Michigan

Ann Arbor, MI 48109, Michigan 48109-2136, USA

DOI: 10.1002/adma.201001761 the widespread implementation of NIL is the limited availability of high-quality molds. High-resolution imprint molds are usually fabricated by slow, serial patterning techniques such as electron-beam (e-beam) lithography. While the NIL tools themselves are relatively inexpensive, the fabrication of highresolution $1 \times$ molds can be prohibitive in terms of the fabrication time and cost. Furthermore, the notion of stamping these expensive master templates into contact with a resist material under high-temperature and elevated pressure radiation raises concerns regarding template durability. Ideally they should be cheap, simple, and easy to replace.

There are several critical properties, in addition to patternability, that must be achieved when designing NIL molds. In the thermal embossing form of NIL, ${ }^{[1,2]}$ a hard mold is pressed into a molten polymer film at elevated temperature and a squeezeflow process generates the pattern. The NIL mold material needs to have appropriate mechanical properties to resist deformation under the imprinting conditions and to withstand the elevated temperatures. With the UV forms of NIL, ${ }^{[3,4]}$ a monomeric liquid flows into the cavities of the mold and is crosslinked into a rigid pattern upon exposure to the radiation. Here, the mold must not only be mechanically robust, but also UV transparent. In all forms of NIL, a low-energy surface is critical to facilitate the release of the high surface area mold from the imprint; the adhesive forces between mold and the template can become significant.

A range of materials has been considered for making NIL molds, varying with the mechanism by which the pattern is generated. The different types of materials used today can be broadly categorized into two classes. ${ }^{[11-13]}$ The first is the soft organic or polymeric materials such as poly(dimethylsiloxane) (PDMS), ${ }^{[14]}$ Teflon, ${ }^{[15]}$ polyurethane ${ }^{[16]}$ or perfluoropolyether (PFPE), ${ }^{[17]}$ while the second includes the hard metal or ceramic stamps such as quartz, $\mathrm{Ni}, \mathrm{Si}$, and $\mathrm{SiO}_{2}$. The soft materials have the advantage that they can be readily fabricated into molds using simple processes, but often run into problems when trying to pattern features smaller than $100 \mathrm{~nm}$ due to dimensional instabilities and a propensity for the features to collapse or distort under load. ${ }^{[14]}$ There have been significant improvements in soft-imprint technologies based on the $h$-PDMS technology, ${ }^{[18-20]}$ but there are still limitations related to mold swelling when trying to pattern materials that contain an organic solvent and shrinkage and/or distortion issues when imprinting at high temperatures and pressures. The transition from soft to hard NIL mold materials addresses these issues and enables high-resolution patterning over large areas, ${ }^{[21,22]}$ but also significantly increases the technical complexity and cost of making the mold. The patterning of hard materials, 
such as $\mathrm{Si}$ or quartz, requires clean room processes that are time-consuming, costly, and cumbersome. A route to low-cost, solution-processable, high-performance imprint molds would critically enable this community.

Cubic silsesquioxanes (SSQs) with the formula $\left[\mathrm{RSiO}_{1.5}\right]_{8}$ are organic-inorganic hybrid materials that consist of a nanometersized, siloxane cube (diameter $\approx 0.5 \mathrm{~nm}$ ) with eight $\mathrm{Si}$ atoms at the vertices, connected through $\mathrm{O}$ atoms at the edges of the cube that can be functionalized by various organic pendant groups, $\mathrm{R}$, via hydrosilylation reactions on the corners of the cube. ${ }^{[23,24]}$ While the inorganic core provides mechanical stiffness and thermal stability, the organic pendants provide solubility in organic solvents that enables solution processing. By varying the composition and length of the organic pendants, one can tune the structure and properties of these SSQ-based materials. A range of nanocomposites ${ }^{[25]}$ and nanoporous materials can be achieved with applicability in a range of technologies including low-dielectric constant-interlayer dielectrics for semiconductor interconnects, ${ }^{[26]}$ hydrogen storage materials, encapsulants for drug delivery, porous media for trapping pollutants and other condensates, and functional coatings for scratch resistance or diffusion barriers. ${ }^{[27]}$ Elsewhere some of the coauthors have developed a series of low-cost bifunctional (having two types of organic pendants $\mathrm{R}$ on the same inorganic cage core) SSQs from rice hull ash, a renewable, abundant agricultural waste product, providing a sustainable route for these versatile materials. ${ }^{[27]}$

Here, we demonstrate that these low-cost, readily available bifunctional SSQs can be engineered into high-performance imprint mold materials that combine the ease of processing found in the soft organic templates with the thermal resistance, structural rigidity, and patterning resolution of the hard template materials. These hybrids offer superior performance as a NIL mold material compared to their wholly organic or inorganic analogs. The fact that they are derived from a natural agricultural waste product is attractive as a green alternative to traditional silicon nanofabrication processes that are energy intensive and utilize many toxic chemicals and gases that are harsh on the environment. This work builds off our previous reports showing that NIL methods can be used to directly pattern SSQ-type material. ${ }^{[5-7]}$ Here, we make the critical leap to show that these cubic-SSQ monomers cannot only be patterned by NIL, but also engineered into high-modulus structures with very little shrinkage, excellent thermal stability, quartz-like transparency in the UV range, and an intrinsically low surface energy. For these reasons they exhibit superior performance as mold replication materials, creating high-modulus replicas of a NIL master that can then be used directly as a robust daughter mold for NIL.

The chemical formulas for the three different SSQs used in this study are shown in Figure 1. The octameric SSQ cores are decorated with various organic groups, many of them terminated with $\mathrm{Si}\left(\mathrm{OC}_{2} \mathrm{H}_{5}\right)_{3}\left[\mathrm{Si}(\mathrm{OEt})_{3}\right]$ functional end groups. Under acidic conditions, the (Si-OEt)s are converted into reactive silanols $(\mathrm{Si}-\mathrm{OH})$, which condense at elevated temperatures into siloxane linkages, forming a cross-linked organosilicate network. The three different SSQ precursors in Figure 1 were designed to establish structure-property relationships. Previously, we have reported that maximizing the number of closed-cage structures in the cross-linked network is important for enhancing mechanical properties like modulus or hardness; the molecules depicted in Figure 1 embrace this concept. The octa(triethoxysilylethyl)(octadimethylsiloxy) octasilsesquioxane (OTSE) is designed to maximize the number of functional end groups (24, as shown in Figure 1a) and, thus, the cross-link density of the organosilicate network. The (b)[tetra(triethoxysilylethyl)dimethylsiloxy](tetradimethylsiloxy) octasilsesquioxane (TTSE) and (c) [tetra(triethoxysilylethyl)dimethylsiloxy][tetra(2-hydroxyethoxyethyldimethylsiloxy] octasilsesquioxane (TOETSE) are bifunctional monomers that contain two distinctly different kinds of pendant groups. ${ }^{[26]}$ In the TTSE case, four out of eight cage vertices are terminated by the $\mathrm{Si}(\mathrm{OEt})_{3}$ functional groups, while the remaining four are terminated by hydrolytically stable $\mathrm{Si}-\mathrm{H}$ bonds [Figure 1b]. The TOETSE contains the same four functional end groups as the TTSE, but the other four corners are (a)

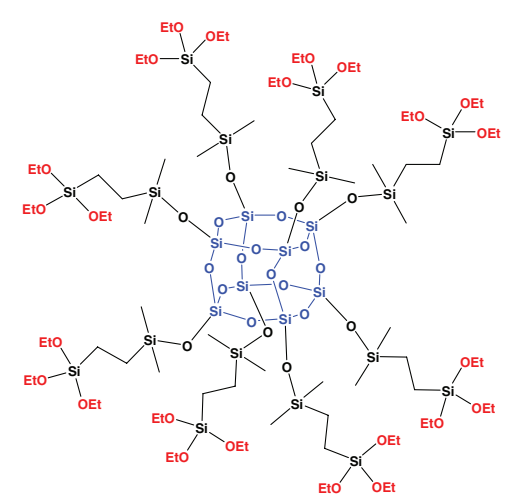

(b)

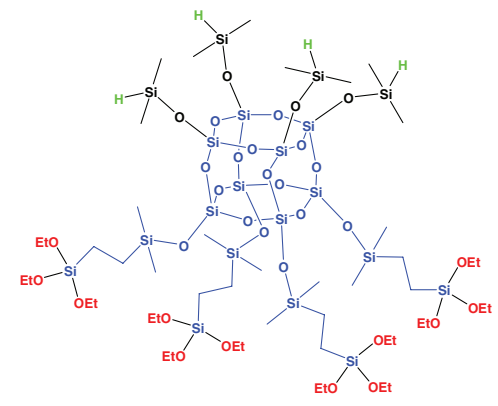

(c)

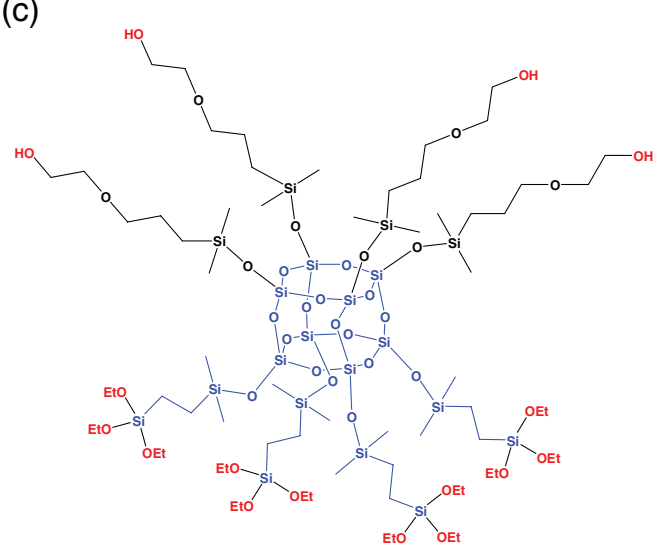

Figure 1. Chemical structures of a) octa(triethoxysilylethyl) (octadimethylsiloxy)octasilsesquioxane (OTSE), b) [tetra(triethoxysilylethyl)dimethylsiloxy] (tetradimethylsil-oxy)octasilsesquioxane (TTSE), and c) [tetra(triethoxysilylethyl)dimethylsiloxy][tetra(2-hydroxy-ethoxyethyldimethylsiloxy]octasilsesquioxane (TOETSE). 
comprised of longer, flexible organic tethers with hydroxyl end groups [Figure 1c].

The most fundamental requirement for a NIL mold material is the ability to fabricate high-resolution patterns in it. Previously, we reported that the high-quality nanoscale patterns can be generated in spin-on organosilicates via one-step thermal NIL process. ${ }^{[6,7]}$ Our original focus for this work was to demonstrate their utility as directly patternable ultralow-k ILDs for next generation microelectronic devices. Here, we show that the organosilicate materials described in Figure 1 can also be patterned with high resolution via the same one-step thermal NIL process, but then used directly as secondary NIL molds to perform subsequent patterning. As we characterize in the Supporting Information, these materials can provide a reasonably high modulus, on the order of $5 \mathrm{GPa}$, a low coefficient of thermal expansion (CTE), on the order of $25 \times 10^{-6}{ }^{\circ} \mathrm{C}^{-1}$, which approaches natural $\mathrm{SiO}_{x}$, the quartz-like transparency in the UV spectrum that is required for NIL patterning, and intrinsically hydrophobic surfaces with water contact angles over $100^{\circ}$, which facilitate pattern release. These are all critical properties for NIL mold applications.

The patterns are directly imprinted into the as-cast SSQ films using a silicon oxide master as the initial NIL template. Choosing the proper imprinting temperature is critical to achieving a high-quality imprint. The secondary condensation of the Si-OH groups in the as-cast film will start when the film is heated. The imprint temperature must be high enough to partially cross-link the pattern and maintain its shape after imprinting when the free-standing pattern is vitrified at much higher temperatures. If the degree of cross-linking during the NIL step is insufficient, the free-standing pattern will start to flow during the hightemperature annealing process. If the imprint temperature is too high, the material will cross-link too much before the imprint process is complete, inhibiting flow of the material into the mold. Here, the imprint temperature of $170{ }^{\circ} \mathrm{C}$ was selected to balance these two competing effects. Once the material flow has occurred and the mold cavities are filled, additional crosslinking in the mold is probably advantageous in terms of pattern stability. As shown in Figure S2a (Supporting Information), the surface energy of the material also decreases with conversion. This means that longer imprint times will help further facilitate the separation of the oxide master from the SSQ imprint.

We now seek to quantify the performance of these SSQ materials as secondary NIL mold replicas. Specular X-ray reflectivity (SXR) can be used to nondestructively quantify the pattern height, the residual layer thickness, and the line-to-space ratio of parallel line spaced gratings patterns. ${ }^{[28,29]}$ Figure 2a shows the (a)

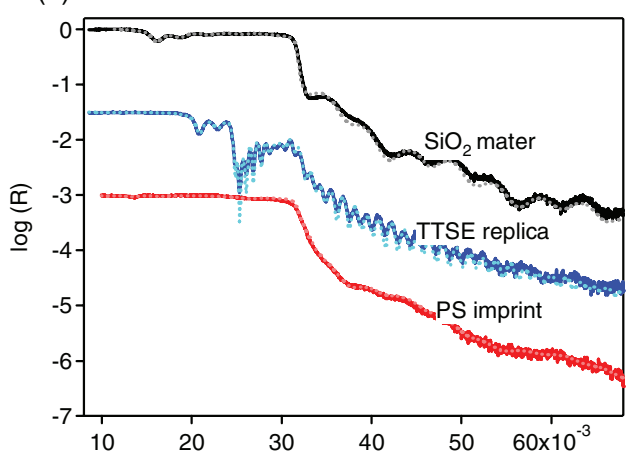

(c)
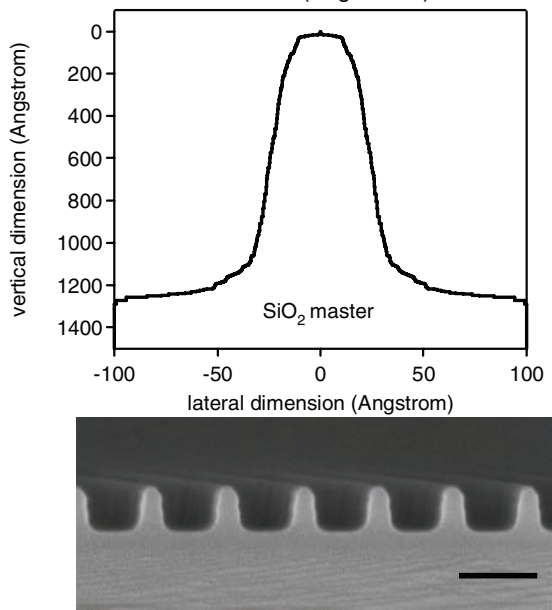

(b)

\begin{tabular}{|c|c|c|}
\hline Sample & pattern height $(\mathrm{nm})$ & line-to-space ratio \\
\hline SiO2 - mother mold & 123.1 & $1: 2.61$ \\
TTSE -daughter mold & 104.6 & $2.26: 1$ \\
PS - imprint & 107.5 & $1: 2.24$ \\
\hline
\end{tabular}

(d)

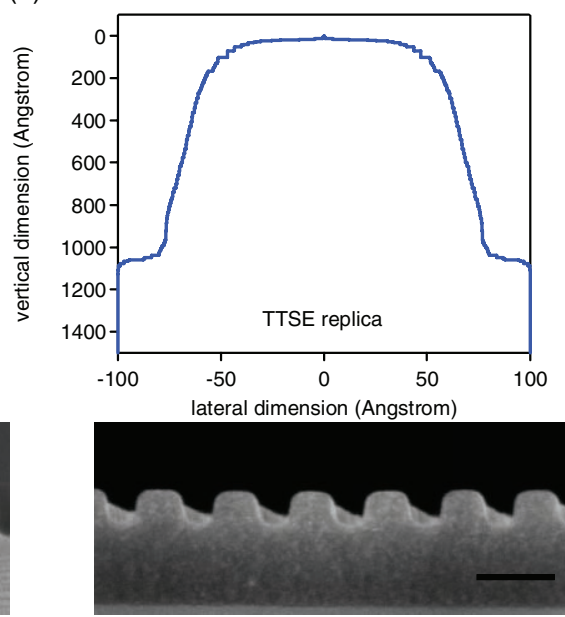

(e)
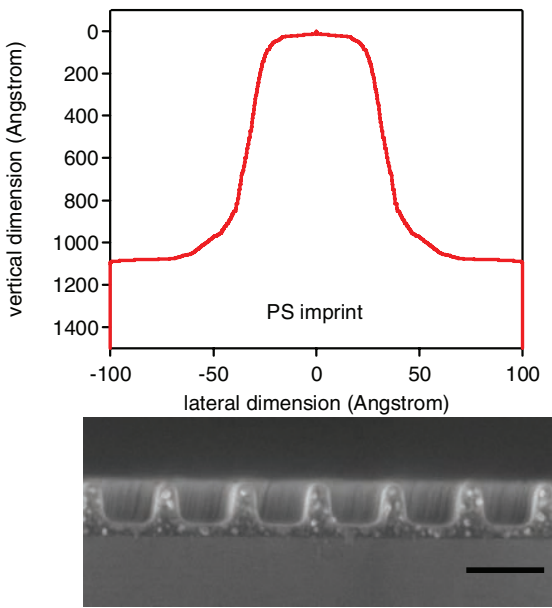

Figure 2. Specular X-ray reflectivity curves are shown in part a) for the master $\mathrm{SiO}_{2}$ mold, the TTSE replica, and the PS imprint from TTSE replica. The fitting results are drawn for each of the experimental curves. The pattern heights and line-to-space ratios from SXR are summarized in part b). Crosssectional pattern shapes deduced from density-depth profiles obtained from the fitting results of SXR, are drawn for c) the master $\mathrm{SiO}_{2} \mathrm{mold}$, d) the TTSE replica, and e) the PS imprint from TTSE replica. The cross-sectional FE-SEM images of each pattern are also shown (scale bar: $200 \mathrm{~nm}$ ). 
SXR data for the master $\mathrm{SiO}_{2}$ mold, the TTSE replica after full vitrification of the patterns, and the polystyrene (PS) imprint made from TTSE replica. Previously we described how these reflectivity curves are fit with a multilayer recursive algorithm based on the formalism of Parratt. ${ }^{[30,31]}$ The fitted models quantify the density profile as a function of distance through the patterned region, the residual layer, and into the supporting substrate. This directly quantifies the residual layer thickness, pattern height, and line-to-space ratio as a function vertical height through the patterns. Although SXR alone cannot determine an absolute length scale for the lateral direction independently, we use the pattern pitch as determined by critical dimension small angle X-ray scattering (CD-SAXS) ${ }^{[28]}$ to convert the line-to-space ratios into the average pattern width as a function of pattern height. The combination of SXR and CD-SAXS can provides the exact pattern cross-section as shown in Figure 2c, $d$, and e for the original $\mathrm{SiO}_{2}$ mold, a TTSE imprint, and a thermal imprint into PS made with the TTSE pattern, respectively. Cross-sectional images from field-emission scanning electron microscopy (FE-SEM) are shown directly below each quantitative pattern profile, showing excellent agreement between the SXR and CD-SAXS data and the real-space images.

The fidelity of the patterning process can be precisely quantified by directly comparing the cross-sectional profiles of the mold and the imprint as shown in Figure 3. Part (a) compares the profiles of the silicon oxide master to the as-imprinted TTSE pattern (before vitrification), revealing a small amount of shrinkage relative to the mold in both the vertical and lateral directions. We previously observed this type of shrinkage on imprinting in similar SSQ-type organosilicate materials, and believe that it is induced by a combination of partial secondary cross-linking and the elimination of residual spin casting solvent during the imprint process. ${ }^{[6,7]}$ This shrinkage is both minimal and highly-reproducible, such that high-quality replicas are easily generated. Unlike our previous observations, almost no shrinkage is observed during the vitrification of the free-standing OTSE and TTSE patterns. While a full explanation of this observation is beyond the scope of this paper, we believe this reflects the higher silanol content of these cubic SSQ monomers compared with our previous non-cubic SSQ oligomers. It has been reported that the onset temperature for secondary condensation depends on the concentration of $\mathrm{Si}-\mathrm{OH}$ groups, ${ }^{[32]}$ with greater silanol contents accelerating the cross-linking reaction kinetics. A greater extent of initial crosslinking at lower temperatures (i.e., during the imprint process) leads to reduced shrinkage during the high-temperature vitrification. ${ }^{[33]}$ This suggests that the degree of cross-linking during the NIL process in both OTSE and TTSE patterns is not only sufficient for the patterns to maintain their free-standing structures, but also high enough to minimize the additional shrinkage that may occur during vitrification.

The next step is to use the TTSE replica to directly imprint a PS film at high temperature and pressure. The profiles of the TTSE mold and the imprinted PS pattern are overlaid in Figure 3b, showing excellent fidelity of the pattern transfer. These imprints are performed without applying a mold release or fluorinated self-assembled monolayer (SAM) to the TTSE patterns. Attempts to directly imprint the high-energy $\mathrm{SiO}_{2}$ patterns into PS without using a fluorinated release coating would have sheared the patterns off during the mold separation. We have performed more than 20 imprints of this TTSE mold into polymeric resists, including PS or PMMA, and observe highquality imprints without degradation. We have also prepared TTSE patterns on a quartz substrate for use as a secondary mold for the UV form of NIL. Using this template we were able to generate patterns of the same quality as Figure 3b (results not shown here) in a commercial UV imprint resist formulation (NX-2010, Nanonex Inc. New Jersey, USA. (Certain commercial materials and equipment are identified in this paper in order to specify adequately the experimental procedure. In no case does such identification imply recommendation by the National Institute of Standards and Technology nor does it imply that the material or equipment identified is necessarily the best available for this purpose.) These results demonstrate that the TTSE and OTSE materials are high-performance materials for creating secondary or daughter NIL templates.

The imprinted patterns in Figure 3 had average line widths of approximately $70 \mathrm{~nm}$. To push the resolution limits of this mold replication strategy, we decreased the line widths of the $\mathrm{SiO}_{2}$ master by wet etching in a buffered oxide solution to obtain approximately $30 \mathrm{~nm}$ line widths with a $20 \mathrm{~nm}$ height, while still maintaining a periodicity of $200 \mathrm{~nm}$. The same procedure was repeated to produce TTSE replicas which were then directly imprinted into poly(methyl methacrylate) (PMMA). Compared to PS, PMMA has a greater surface energy and should therefore be more prone to mold adhesion issues. Once again, (a)

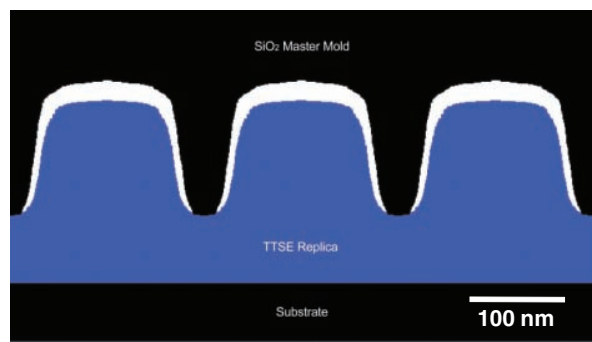

(b)

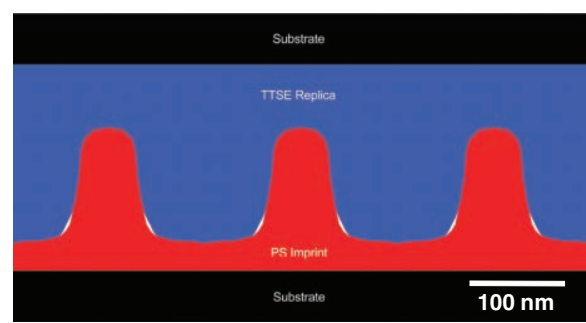

Figure 3. Direct comparison of the mold and imprint shape for a) NIL of TTSE using a $\mathrm{SiO}_{2}$ master mold and b) NIL of PS using TTSE replica. 
(a)

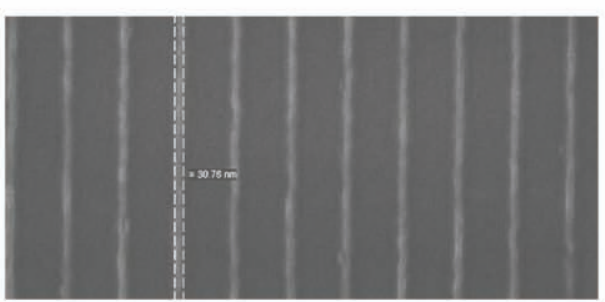

(b)

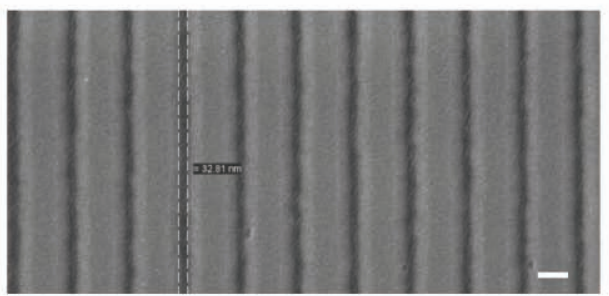

(c)

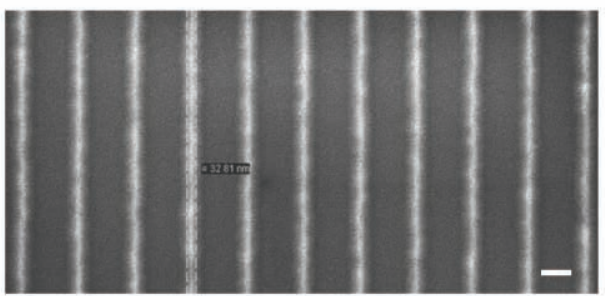

(d)

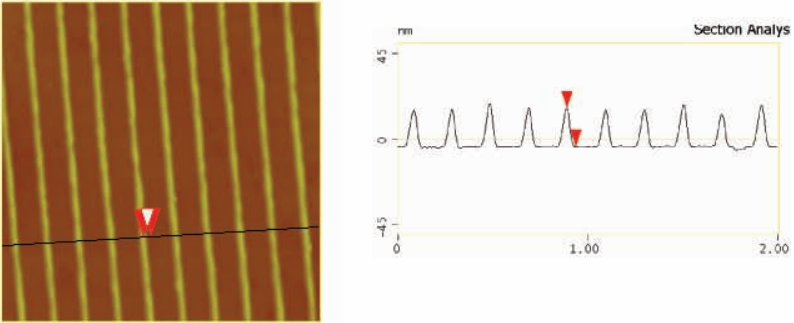

(e)

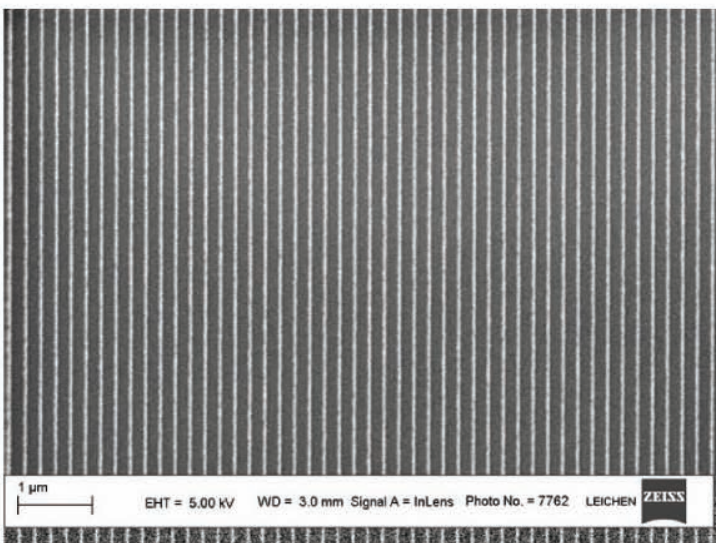

Figure 4. Top-down FE-SEM images for a) master $\mathrm{SiO}_{2}$ grating pattern with an approximately $30 \mathrm{~nm}$ line width and $20 \mathrm{~nm}$ pattern height. b) TTSE replica of the master $\mathrm{SiO}_{2}$ mold, and c) PMMA imprint from the TTSE replica. d) AFM height images $(2 \mu \mathrm{m} \times 2 \mu \mathrm{m})$ for the PMMA imprint is shown, combined with cross-sectional analysis data, which shows that the pattern height is about $20 \mathrm{~nm}$. The uniform PMMA imprint from TTSE replica shown in part e) indicates that the secondary PMMA pattern is uniform in the large area without noticeable defect.

high-quality patterns with line or trench widths of approximately $30 \mathrm{~nm}$ were faithfully transferred through the process as shown in Figure $4 \mathrm{a}-\mathrm{c}$. For these thin lines on a large periodicity, the SXR and CD-SAXS measurement methods lose their sensitivity, making it difficult to quantify fidelity of the patterning process. The qualitative SEM images in Figure 4a-c indicate, however, that the pattern quality in the PMMA imprint is nominally the same as the $\mathrm{SiO}_{2}$ master and the replication process does not appear to degrade the resolution. This is consistent with the high-quality line profile obtained by AFM in Figure $4 \mathrm{~d}$ for the imprinted PMMA pattern. A lower magnification image of the imprinted PMMA patterns indicates that uniform patterns are generated over large areas without noticeable defect, as shown in Figure 4e. In the Supporting Information, we provide preliminary qualitative evidence to suggest that the patterning resolution of these materials can be extended to below $10 \mathrm{~nm}$ by continued wet etching of the silicon oxide mold, but the quality of the mold in these cases is significantly deteriorated.

In conclusion, we have precisely quantified the performance of octameric organosilicate materials with tunable chemical and mechanical properties as replication materials to create low-cost, high-resolution secondary nanoimprint lithography molds. Our measurements show that these materials can be tuned to exhibit several of the critical properties requisite for secondary imprint mold applications. They can be engineered with Young's moduli of over 4.5 GPa (OTSE: $5.78 \pm 0.161 \mathrm{GPa}$, TTSE: $4.61 \pm 0.088 \mathrm{GPa}$ (The error bars presented throughout this manuscript indicate the relative standard uncertainty of the measurement.) to resist mechanical distortions under imprint, provide optical transmittance comparable to quartz in the UV spectrum, display low CTEs and excellent thermal stability for high-temperature imprinting, and offer intrinsically low surface energies to facilitate separation of the mold from the imprint. Using these materials, we can fabricate high-resolution replicas of $\mathrm{SiO}_{2}$ imprint masters and then directly use these replicas to produce high-quality imprints via both UV and thermal NIL, with a patterning resolution approaching just a few nanometers. These organosilicate materials are derived from a naturally abundant agricultural waste product, providing a simplified, straightforward, and cost-effective alternative to conventional silicon patterning methods to produce nanoimprint lithography templates.

\section{Experimental Section}

Hydrolysis Reaction of SSQs: The SSQ monomers (0.1 to $10.0 \mathrm{mmol}$ ) were added to a stirred tetrahydrofuran (THF, $6 \mathrm{~mL}$ ) solution in a $50 \mathrm{~mL}$ round bottom flask. To this, a mixture of distilled water $\left(\mathrm{H}_{2} \mathrm{O}\right)$ and hydrochloric acid $(\mathrm{HCl}, 1 \mathrm{M})$ was added drop wise while stirring at $0^{\circ} \mathrm{C}$. The molar ratio of the reaction mixture was 1:0.2:50 [SSQs: $\mathrm{HCl}: \mathrm{H}_{2} \mathrm{O}$ ]. 
After stirring the reaction mixture for 0.2 to $10.0 \mathrm{~h}$ at room temperature, the reaction mixture was poured into a solvent system consisting of diethylether and water, resulting in a phase separation. The organic phase was recovered and stirred with magnesium sulfate $\left(\mathrm{MgSO}_{4}\right)$ for $30 \mathrm{~min}$ followed by filtering through $0.4 \mu \mathrm{m}$ PTFE syringe filter. Solvent was slowly evaporated using a rotary evaporator. The remaining product was dissolved in either propylene glycol propyl ether (PGPE) or propylene glycol monomethyl ether acetate (PGMEA) as the spin-casting solution and spun into $300 \mathrm{~nm}$ thick films.

Thermalgravimetric Analysis (TGA): TGA measurements were performed on a TGA Q500 (TA instrument, Delaware, USA) with the sample in an inert nitrogen atmosphere. The samples were dried at $60{ }^{\circ} \mathrm{C}$ under vacuum prior to testing, and then thermally ramped in the TCA to $500^{\circ} \mathrm{C}$ at a heating rate of $10^{\circ} \mathrm{C} \mathrm{min}^{-1}$.

Nanoindentation: Depth Sensing Indentation (DSI) measurements were conducted using the Dynamic Contact Module (DCM) on a MTS Nanolndenter. All films were made thicker than $500 \mathrm{~nm}$ in order to eliminate substrate effect. Indentations were made to a depth of $100 \mathrm{~nm}$ using a pyramidal Berkovich tip. The contact stiffness between the sample and the indenter was measured as a function of indentation depth by imposing a $2 \mathrm{~nm}, 75 \mathrm{~Hz}$ oscillation over the programmed loading. ${ }^{[34]}$ The loading was conducted at a constant strain rate of $0.05 \mathrm{~s}^{-1}$. The average modulus and hardness were determined for each indent over a depth range of 25 to $50 \mathrm{~nm}$. The reported average modulus and hardness were determined from an average of 20 indents into each film.

Contact Angle Measurement: Contact angle measurements were made by the sessile drop technique using a Krüss $G 2$ contact-angle instrument with the sample held at room temperature. SSQ films were first annealed at different temperature (as-cast, 100,170 , and $380^{\circ} \mathrm{C}$ ) under nitrogen environment before the contact angle measurements. A deionized (DI) water droplet (approximately $2 \mathrm{~mL}$ in volume) was deposited on the sample surface and the static equilibrium contact angle (CA) was measured immediately upon needle removal. The average of at least five measurements taken at different positions on each sample was reported as the contact angle.

UV-vis Spectrophotometry: UV-vis was performed in transmission mode on a PerkinElmer Lambda 950 UV-vis-NIR spectrophotometer over the range of 1000 to $185 \mathrm{~nm}$. Data was recorded in $1 \mathrm{~nm}$ increments with an instrument integration time of at least $0.12 \mathrm{~s}$ per increment. The reference beam was left unobstructed for these measurements and transmission of the appropriate bare substrate was substracted from the reported data.

Buffered Oxide Etching (BOE) of Master $\mathrm{SiO}_{2}$ Mold: The $\mathrm{SiO}_{2}$ master mold was a periodic silicon oxide line-space gratings structure, with approximately $100 \mathrm{~nm}$ line widths on a $200 \mathrm{~nm}$ pitch and $170 \mathrm{~nm}$ line heights. To vary the dimension of the master mold, buffered oxide etch solution (BOE, $\mathrm{NH}_{4} \mathrm{~F}: \mathrm{HF}=6: 1$ ) was used with different times followed by $\mathrm{H}_{2} \mathrm{O}$ washing and $\mathrm{N}_{2}$ blowing.

Direct thermal NIL of the SSQs: SSQ films were prepared from PGPE or PGMEA solution by spin coating at $157 \mathrm{rad} \mathrm{s}^{-1}$ (1500 rpm) for $20 \mathrm{~s}$. Then, films were subjected into a conformal contact with the $\mathrm{SiO}_{2}$ molds using an imprint machine (NX-2000, Nanonex Inc.). After evacuating the sample chamber for $2 \mathrm{~min}$, the imprints were made in two steps: $10 \mathrm{~s}$ at $100^{\circ} \mathrm{C}$ under a pressure of $1.4 \mathrm{MPa}$, followed by an additional $3 \mathrm{~min}$ at $170^{\circ} \mathrm{C}$ under a pressure of $3.4 \mathrm{MPa}$. The imprint tool then was cooled to $55^{\circ} \mathrm{C}$ before releasing the pressure and separating the pattern from the mold. The SSQ imprints were further vitrified at $400{ }^{\circ} \mathrm{C}$ under $\mathrm{N}_{2}$ environment for $1 \mathrm{~h}$ with ramping rate of $3{ }^{\circ} \mathrm{C} \mathrm{min}-1$. NIL was used to directly transfer patterns into as-cast films, before thermal vitrification, using a series of $\mathrm{SiO}_{2}$ master molds described above. All the master $\mathrm{SiO}_{2}$ patterns were treated with a low-energy SAM deposited from tridecafluoro-1,1,2,2 (tetrahydrooctyl) trichlorosilane vapor to facilitate mold release.

Thermal NIL of polymeric materials: The imprints were made into poly(styrene) (PS, $18.7 \mathrm{~kg} \mathrm{~mol}^{-1}$ ) or poly(methyl methacrylate) (PMMA, $17.9 \mathrm{~kg} \mathrm{~mol}^{-1}$ ) standards (obtain from Polymer Laboratories). PS was dissolved in toluene and PMMA was dissolved in anisole with concentrations of $6.7 \%$ and $10 \%$ by mass, respectively. The films were spin-cast at $209 \mathrm{rad} \mathrm{s}^{-1}(2000 \mathrm{rpm})$ onto Si wafers with a native oxide surface. The spin-cast films were baked under vacuum for $1 \mathrm{~h}$ at $150{ }^{\circ} \mathrm{C}$ to remove the residual solvent before imprinting. The imprints were made on a NX-2000 imprint tool with OTSE or TTSE imprints consisted of parallel line-and-space grating structures. The OTSE and TTSE imprints were used directly as secondary molds without the deposition of a low surface energy release layer. After sufficiently evacuating the sample chamber, the imprints were made in two steps: $10 \mathrm{~s}$ at $100^{\circ} \mathrm{C}$ and $1.4 \mathrm{MPa}$, followed by $3 \mathrm{~min}$ at $150^{\circ} \mathrm{C}$ and $3.4 \mathrm{MPa}$. The imprint tool then was cooled to $55{ }^{\circ} \mathrm{C}$, below the glass transition temperature of the resist, before releasing the pressure and separating the pattern from the mold.

Specular X-ray Reflectivity (SXR): SXR measurements were performed on a Philips X'PERT diffractometer using $\mathrm{Cu}-K_{\alpha}$ X-ray radiation $(\lambda=$ $1.54 \AA)$. The incident beam was focused with a curved mirror into a 4 bounce Ge [220] crystal monochromator before being incident onto the sample. The reflected beam was further conditioned with a 3-bounce $\mathrm{Ge}$ [220] crystal monochromator ensure the specular condition. The angular reproducibility of the goniometers that control the sample rotation and angular position of $\mathrm{X}$-ray detector was $0.0001^{\circ}$. The reflectivity was collected at $25{ }^{\circ} \mathrm{C}$ under vacuum for all samples.

Precision out-of-plane CTE measurements were made by measuring the thickness of the films as a function of temperature using SXR. The thickness of a film was determined from the spacing of the interference fringes. Planar SSQ films were placed in a specially designed vacuum chamber and the thickness was measured at four different temperatures; $25,75,125$, and $175^{\circ} \mathrm{C}$. The reflectivity measurements were performed in a vacuum of $1 \times 10^{-6} \mathrm{~Pa}$ after annealing the film at the predetermined temperature for $1 \mathrm{~h}$. After the measurement at $175^{\circ} \mathrm{C}$, the samples were cooled back to $25^{\circ} \mathrm{C}$ and the measurements were repeated in order to confirm a reversible expansion and contraction behavior.

Field-Emission Scanning Electron Microscopy (FE-SEM): The crosssection surface was prepared by cleaving the sample perpendicular to the grating direction at room temperature. The freshly cut surface was then coated with less than $5 \mathrm{~nm}$ Au/Pd by Denton Desk $1 \mathrm{~V}$ sputter machine to reduce surface charge accumulation in the electric field. The specimens were analyzed with a field emission scanning electron microscope (Zeiss Ultra-60) at room temperature. The accelerating voltage was set to $5 \mathrm{keV}$ and the working distance set to between 5 and $8 \mathrm{~mm}$.

\section{Supporting Information}

Supporting Information is available from the Wiley Online Library or from the author.

\section{Acknowledgements}

The authors acknowledge the financial support from the NIST Office of Microelectronics Programs. We also acknowledge the Nanofabrication Laboratory of the Center for Nanoscale Science and Technology (CNST) in NIST for providing facilities. Work done at Mayaterials was supported through a Phase I SBIR award from the US Army Soldier Systems Center (Natick, MA) through contract W911QY-07-C-0012. This article is part of a Special Issue on Materials Science at the National Institute of Standards and Technology (NIST).

Received: May 12, 2010

Published online: August 16, 2010

[1] S. Y. Chou, P. R. Krauss, P. J. Renstrom, Appl. Phys. Lett. 1995, 67, 3114.

[2] S. Y. Chou, P. R. Krauss, P. J. Renstrom, Science 1996, 5, 85

[3] J. Haisma, M. Verheijen, K. van den Heuvel, J. van den Berg, J. Vac. Sci. Technol. B. 1996, 14, 4124. 
[4] S. J. Colburn, M. Stewart, S. Damle, T. Bailey, B. Choi, M. Wedlake, T. Michaelson, S. V. Sreenivasan, J. Ekerdt, C. G. Willson, SPIE Proc. 1999, 3676, 379.

[5] G. M. Schmid, M. D. Stewart, J. Wetzel, F. Palmieri, J. Hao, Y. Nishimura, K. Jen, E. K. Kim, D. J. Resnick, A. Liddle, C. G. Wilson, J. Vac. Sci. Technol. B. 2006, 24, 1283.

[6] H. W. Ro, R. L. Jones, H. Peng, D. R. Hines, H.-J. Lee, E. K. Lin, A. Karim, D. Y. Yoon, D. W. Gidley, C. L. Soles, Adv. Mater. 2007, 19, 2919.

[7] H. W. Ro, H. Peng, K.-i. Niihara, H.-J. Lee, E. K. Lin, A. Karim, D. W. Gidley, H. Jinnai, D. Y. Yoon, C. L. Soles, Adv. Mater. 2008, 20, 1934.

[8] B. D. Terris, T. Thomson, J. Phys. D: Appl. Phys. 2005, 38, R199.

[9] J.-S. Wi, H.-S. Lee, K. Lim, S.-W. Nam, H.-M. Kim, S.-Y. Park, J. J. Lee, C. D. Hong, S. Jin, K.-B. Kim, Small 2008, 4, 2118.

[10] K.-J. Byoen, S.-Y. Hwang, H. Lee, Appl. Phys. Lett. 2007, 91, 0911061.

[11] B. D. Gates, Q. Xu, M. Stewart, D. Ryan, C. G. Willson, G. M. Whitesides, Chem. Rev. 2005, 105, 1171.

[12] Z. Nie, E. Kumacheva, Nat. Mater. 2008, 7, 277.

[13] L. J. Guo, J. Phys. D: Appl. Phys. 2004, 37, R123.

[14] Y. Xia, G. M. Whitesides, Angew. Chem. Int. Ed. 1998, 37, 550.

[15] D.-Y. Khang, H.-H. Lee, Adv. Mater. 2004, 16, 176.

[16] Y. Xia, J. J. McClelland, R. Gupta, D. Qin, X.-M. Zhao, L. L. Sohn, R. J. Celotta, G. M. Whitesides, Adv. Mater. 1997, 9, 147.

[17] J. P. Rolland, E. C. Hagberg, G. M. Denison, K. R. Carter, J. M. DeSimone, Angew. Chem. Int. Ed. 2004, 43, 5796.

[18] H. Schmid, B. Michel, Macromolecules 2000, 33, 3042.
[19] B. D. Gates, G. M. Whitesides, J. Am. Chem. Soc. 2003, 125, 14986.

[20] F. Hua, Y. Sun, A. Gaur, M. A. Meitl, L. Bilhaut, L. Rotkina, J. Wang, P. Geil, M. Shim, J. A. Rogers, Nano Lett. 2004, 4, 2467.

[21] M. D. Austin, H. Ge, W. Wu, M. Li, Z. Yu, D. Wasserman, S. A. Lyon, S. Y. Chou, Appl. Phys. Lett. 2004, 84, 5299.

[22] H. Gao, H. Tan, W. Zhang, K. Morton, S. Y. Chou, Nano Lett. 2006, $6,2438$.

[23] G. Li, L. Wang, H. Ni, C. U. Pittman, J. Inorg. Organomet. Polym. 2002, 11, 123.

[24] R. M. Laine, J. Mater. Chem. 2005, 15, 3725.

[25] M. Joshi, B. S. Butola, J. Macromol. Sci., Polym. Rev. 2004, 44, 389.

[26] R. Q. Su, T. E. Muller, J. Prochazka, J. A. Lercher, Adv. Mater. 2002, 14, 1369.

[27] R. M. Laine, M. Roll, M. Asuncion, S. Sulaiman, V. Popova, D. Bartz, D. J. Krug, P. H. Mutin, J. Sol-Gel Sci. Technol. 2008, 46, 335.

[28] H.-J. Lee, C. L. Soles, H. W. Ro, R. L. Jones, E. K. Lin, W.-I. Wu, D. R. Hines, Appl. Phys. Lett. 2005, 87, 263111.

[29] H.-J. Lee, H. W. Ro, C. L. Soles, R. L. Jones, E. K. Lin, W.-I. Wu, D. R. Hines, J. Vac. Sci. Technol. B 2005, 23, 3023.

[30] L. G. Parratt, Phys. Rev. 1954, 95, 359.

[31] J. F. Anker, C. F. Majkrzak, SPIE Proc. 1992, 1738, 260.

[32] Q. R. Huang, W. Volksen, E. Huang, M. Toney, C. W. Frank, R. D. Miller, Chem. Mater. 2002, 14, 3676.

[33] H. W. Ro, K.-i. Niihara, L. Ming, H. Jinnai, D. W. Gidley, C. L. Soles, unpublished.

[34] W. C. Oliver, G. M. Pharr, J. Mater. Res. 1992, 7, 1564. 